\title{
Colour classification method for recycled melange fabrics
}

\author{
Rocco Furferi
}

\begin{abstract}
Classification of wasted woollen textiles on the basis of their colour is a basic approach for the supply of a raw material which does not involve the cost of the colouring process. Colour classification is a very difficult task, especially when a fabric is composed by differently coloured fibre (melange fabric). Many systems have been developed in the last years for colour classification of textiles. Unfortunately such colour classification systems are not able to correctly classify melange fabrics. In the present work a method for real-time classification of melange colour woollen fabrics is proposed. The provided approach, that is suitable also for classifying solid colour fabrics, integrates a Machine Vision (MV) system, able to acquire high resolution images, with a clustering algorithm capable of mapping the colour pixel of fabric images into a series of colour classes.

The proposed system provides a colour classification with a misclassification less than $10 \%$ when compared with the classification resulting from a panel of expert human operators. A comparison between the proposed method and some tools stated in scientific literature is also afforded.
\end{abstract}

